\title{
Boundary value problems for fractional differential inclusions with Hadamard type derivatives in Banach spaces
}

\author{
John R. Graef, Nassim Guerraiche and Samira Hamani
}

\begin{abstract}
The authors establish sufficient conditions for the existence of solutions to boundary value problems for fractional differential inclusions involving the Hadamard type fractional derivative of order $\alpha \in(1,2]$ in Banach spaces. Their approach uses Mönch's fixed point theorem and the Kuratowski measure of noncompacteness.
\end{abstract}

Mathematics Subject Classification (2010): 26A33, 34A08, 34A60, 34B15.

Keywords: Fractional differential inclusion, Hadamard-type fractional derivative, fractional integral, Mönch's fixed point theorem, Kuratowski measure of noncompacteness.

\section{Introduction}

In this paper we are concerned with the existence of solutions to boundary value problems (BVP for short) for fractional order differential inclusions. In particular, we consider the boundary value problem

$$
\begin{gathered}
{ }^{H} D^{r} y(t) \in F(t, y(t)), \text { for a.e. } t \in J=[1, T], 1<r \leq 2, \\
y(1)=0, y(T)=y_{T},
\end{gathered}
$$

where ${ }^{H} D^{r}$ is the Hadamard fractional derivative, $(E,|\cdot|)$ is a Banach space, $\mathcal{P}(E)$ is the family of all nonempty subsets of $E, F:[1, T] \times E \rightarrow \mathcal{P}(E)$ is a multivalued map, and $y_{T} \in \mathbb{R}$.

Differential equations of fractional order are valuable in modeling phenomena in various fields of science and engineering. They can be found in viscoelasticity, electrochemistry, control, porous media, electromagnetism, etc. The monographs of Hilfer [18], Kilbas et al. [19], Podlubny [23], and Momani et al. [21] are very good sources on the background mathematics and various applications of fractional derivatives. The literature on Hadamard-type fractional differential equations has not undergone as 
much development as it has for the Caputo and Riemann-Liouville fractional derivatives; see, for example, the papers of Ahmed and Ntouyas [2], Benhamida, Graef, and Hamani [10], and Thiramanus, Ntouyas, and Tariboon [24].

The fractional derivative that Hadamard [16] introduced in 1892 differs from other fractional derivatives in the sense that the kernel of the integral in the definition of the Hadamard derivative contains a logarithmic function with an arbitrary exponent. A detailed description of the Hadamard fractional derivative and integral can be found in $[11,12,13]$.

In this paper, we present existence results for the problem (1.1)-(1.2) in the case where the right hand side is convex valued. This result relies on the set-valued analog of Mönch's fixed point theorem combined with the technique of measure of noncompactness. Recently, this has proved to be a valuable tool in studying fractional differential equations and inclusions in Banach spaces; for additional details, see the papers of Laosta et al. [20], Agarwal et al. [1], and Benchohra et al. [7, 8, 9]. Our results here extend to the multivalued case some previous results in the literature and constitutes what we hope is an interesting contribution to this emerging field. We include an example to illustrate our main results.

\section{Preliminaries}

This section contains definitions, concepts, lemmas, and preliminary facts that will be used in the remainder of this paper. Let $C(J, E)$ be the Banach space of all continuous functions from $J$ into $E$ with the norm

$$
\|y\|_{\infty}=\sup \{|y(t)|: t \in J\},
$$

and let $L^{1}(J, E)$ be the Banach space of Lebesgue integrable functions $y: J \rightarrow E$ with the norm

$$
\|y\|_{L^{1}}=\int_{1}^{T}|y(t)| d t .
$$

The space $A C^{1}(J, E)$ is the space of functions $y: J \rightarrow E$ that are absolutely continuous and have an absolutely continuous first derivative.

For any Banach space $X$, we set

$$
\begin{aligned}
& P_{c l}(X)=\{Y \in \mathcal{P}(X): Y \text { is closed }\} \\
& P_{b}(X)=\{Y \in \mathcal{P}(X): Y \text { is bounded }\} \\
& P_{c p}(X)=\{Y \in \mathcal{P}(X): Y \text { is compact }\}, \text { and } \\
& P_{c p, c}(X)=\{Y \in \mathcal{P}(X): Y \text { is compact and convex }\}
\end{aligned}
$$

A multivalued map $G: X \rightarrow \mathcal{P}(X)$ is convex (closed) valued if $G(X)$ is convex (closed) for all $x \in X$. We say that $G$ is bounded on bounded sets if $G(B)=\cup_{x \in B} G(x)$ is bounded in $X$ for all $B \in P_{b}(X)$ (i.e., $\sup _{x \in B}\{\sup \{|y|: y \in G(x)\}\}$ is bounded).

The mapping $G$ is upper semi-continuous (u.s.c) on $X$ if for each $x_{0} \in X$, the set $G\left(x_{0}\right)$ is a nonempty closed subset of $X$, and for each open set $N$ of $X$ containing $G\left(x_{0}\right)$, there exists an open neighborhood $N_{0}$ of $x_{0}$ such that $G\left(N_{0}\right) \subset N$. A map $G$ is said to be completely continuous if $G(B)$ is relatively compact for every $B \in P_{b}(X)$.

If the multivalued map $G$ is completely continuous with nonempty compact values, then $G$ is u.s.c if and only if $G$ has a closed graph (i.e., $x_{n} \rightarrow x_{*}, y_{n} \rightarrow y_{*}$, 
$y_{n} \in G\left(x_{n}\right)$ imply $\left.y_{*} \in G\left(x_{*}\right)\right)$. The mapping $G: X \rightarrow \mathcal{P}(X)$ has a fixed point if there exists $x \in X$ such that $x \in G(x)$. The set of fixed points of the multivalued operator $G$ will be denoted by Fix $G$. A multivalued map $G: J \rightarrow P_{c l}(X)$ is said to be measurable if for every $y \in X$, the function

$$
t \rightarrow d(y, G(t))=\inf \{|y-z|: z \in G(t)\}
$$

is measurable.

Definition 2.1. A multivalued map $F: J \times E \rightarrow \mathcal{P}(E)$ is said to be Carathéodory if:

(1) $t \rightarrow F(t, u)$ is measurable for each $u \in E$;

(2) $u \rightarrow F(t, u)$ is upper semicontinuous for a.e. $t \in J$.

For each $y \in A C^{1}(J, E)$, define the set of selections of $F$ by

$$
S_{F, y}=\left\{v \in L^{1}(J, E): v(t) \in F(t, y(t)) \text { a.e. } t \in J\right\} \text {. }
$$

Let $(X, d)$ be a metric space induced from the normed space $(X,|\cdot|)$. The function $H_{d}: \mathcal{P}(X) \times \mathcal{P}(X) \rightarrow \mathbb{R}_{+} \cup\{\infty\}$ given by

$$
H_{d}(A, B)=\max \left\{\sup _{a \in A} d(a, B), \sup _{b \in B} d(A, b)\right\}
$$

is known as the Hausdorff-Pompeiu metric.

For more details on multivalued maps, see the books of Aubin and Cellina [4], Aubin and Frankowska [5], Castaing and Valadier [14], and Deimling [15].

For convenience, we first recall the definitions of the Kuratowski measure of noncompacteness and summarize the main properties of this measure.

Definition 2.2. ([3,6]) Let $E$ be a Banach space and let $\Omega_{E}$ be the bounded subsets of $E$. The Kuratowski measure of noncompactness is the map $\beta: \Omega_{E} \rightarrow[0, \infty)$ defined by

$$
\beta(B)=\inf \left\{\epsilon>0: B \subset \bigcup_{j=1}^{m} B_{j} \text { and } \operatorname{diam}\left(B_{j}\right) \leq \epsilon\right\} .
$$

Properties: The Kuratowski measure of noncompactness satisfies the following properties (for more details see $[3,6]$ ):

$\left(\mathrm{P}_{1}\right) \quad \beta(B)=0$ if and only if $\bar{B}$ is compact ( $B$ is relatively compact).

$\left(\mathrm{P}_{2}\right) \quad \beta(B)=\beta(\bar{B})$.

$\left(\mathrm{P}_{3}\right) \quad A \subset B$ implies $\beta(A) \leq \beta(B)$.

$\left(\mathrm{P}_{4}\right) \quad \beta(A+B) \leq \beta(A)+\beta(B)$.

$\left(\mathrm{P}_{5}\right) \quad \beta(c B)=|c| \beta(B), \quad c \in \mathbb{R}$.

$\left(\mathrm{P}_{6}\right) \quad \beta(\operatorname{conv} B)=\beta(B)$.

Here $\bar{B}$ and conv $B$ denote the closure and the convex hull of the bounded set $B$, respectively.

For a given set $V$ of functions $u: J \rightarrow E$, we set

$$
V(t)=\{u(t): u \in V\}, t \in J,
$$


and

$$
V(J)=\{u(t): u \in V(t), t \in J\} .
$$

Theorem 2.3. ([17], [22, Theorem 1.3]) Let $E$ be a Banach space and let $C$ be a countable subset of $L^{1}(J, E)$ such that there exists $h \in L^{1}\left(J, \mathbb{R}_{+}\right)$with $|u(t)| \leq h(t)$ for a.e. $t \in J$ and every $u \in C$. Then the function $\varphi(t)=\beta(C(t))$ belongs to $L^{1}\left(J, \mathbb{R}_{+}\right)$ and satisfies

$$
\beta\left(\left\{\int_{0}^{T} u(s) d s: u \in C\right\}\right) \leq 2 \int_{0}^{T} \beta(C(s)) d s .
$$

Lemma 2.4. ([20, Lemma 2.6]) Let $J$ be a compact real interval, $F$ be a Carathéodory multivalued map, and let $\theta$ be a linear continuous map from $L^{1}(J, E) \rightarrow C(J, E)$. Then the operator

$$
\theta \circ S_{F, y}: L^{1}(J, E) \rightarrow P_{c p, c}(C(J, E)), \quad y \rightarrow\left(\theta \circ S_{F, y}\right)(y)=\theta\left(S_{F, y}\right)
$$

is a closed graph operator in $L^{1}(J, E) \times C(J, E)$.

In what follows, $\log (\cdot)=\log _{e}(\cdot)$, and $n=[r]+1$ where $[r]$ denotes the integer part of $r$.

Definition 2.5. ([19]) The Hadamard fractional integral of order $r$ for a function $h$ : $[1,+\infty) \rightarrow \mathbb{R}$ is defined by

$$
I^{r} h(t)=\frac{1}{\Gamma(r)} \int_{1}^{t}\left(\log \frac{t}{s}\right)^{r-1} \frac{h(s)}{s} d s, r>0
$$

provided the integral exists.

Definition 2.6. ([19]) For a function $h$ on the interval $[1,+\infty)$, the Hadamard fractional derivative of $h$ of order $r$ is defined by

$$
\left({ }^{H} D^{r} h\right)(t)=\frac{1}{\Gamma(n-r)}\left(t \frac{d}{d t}\right)^{n} \int_{1}^{t}\left(\log \frac{t}{s}\right)^{n-r-1} \frac{h(s)}{s} d s, n-1<r<n, n=[r]+1 .
$$

Let us now recall Mönch's fixed point theorem.

Theorem 2.7. ([22, Theorem 3.2]) Let $K$ be a closed and convex subset of a Banach space $E, U$ be a relatively open subset of $K$, and $N: \bar{U} \rightarrow \mathcal{P}(K)$. Assume that graph $N$ is closed, $N$ maps compact sets into relatively compact sets, and for some $x_{0} \in U$, the following two conditions are satisfied:

(i) $M \subset \bar{U}, M \subset \operatorname{conv}\left(x_{0} \cup N(M)\right), \bar{M}=\bar{C}$, with $C$ a countable subset of $M$, implies $\bar{M}$ is compact;

(ii) $x \notin(1-\lambda) x_{0}+\lambda N(x)$ for all $x \in \bar{U} \backslash U, \quad \lambda \in(0,1)$.

Then there exists $x \in \bar{U}$ with $x \in N(x)$. 


\section{Main results}

Let us start by defining what we mean by a solution of the problem (1.1)-(1.2).

Definition 3.1. A function $y \in A C^{1}(J, E)$ is said to be a solution of (1.1)-(1.2) if there exist a function $v \in L^{1}(J, E)$ with $v(t) \in F(t, y(t))$ for a.e. $t \in J$, such that ${ }^{H} D^{\alpha} y(t)=v(t)$ on $J$, and the conditions $y(1)=0$ and $y(T)=y_{T}$ are satisfied.

Lemma 3.2. Let $h: J \rightarrow E$ be a continuous function. A function $y$ is a solution of the fractional integral equation

$$
y(t)=\frac{1}{\Gamma(r)} \int_{1}^{t}\left(\log \frac{t}{s}\right)^{r-1} h(s) \frac{d s}{s}+\frac{(\log t)^{r-1}}{(\log T)^{r-1}}\left[y_{T}-\frac{1}{\Gamma(r)} \int_{1}^{T}\left(\log \frac{T}{s}\right)^{r-1} h(s) \frac{d s}{s}\right]
$$

if and only if $y$ is a solution of the fractional $B V P$

$$
\begin{gathered}
{ }^{H} D^{r} y(t)=h(t), \text { for a.e. } t \in J=[1, T], \quad 1<r \leq 2, \\
y(1)=0, y(T)=y_{T} .
\end{gathered}
$$

Proof. Applying the Hadamard fractional integral of order $r$ to both sides of (3.2), we obtain

$$
y(t)=c_{1}(\log t)^{r-1}+c_{2}(\log t)^{r-2}+{ }^{H} I^{r} h(t) .
$$

From (3.3), we have $c_{2}=0$ and

$$
c_{1}=\frac{1}{(\log T)^{r-1}}\left[y_{T}-{ }^{H} I^{r} h(T)\right] .
$$

Hence, we obtain (3.1). Conversely, it is clear that if $y$ satisfies equation (3.1), then (3.2)-(3.3) hold.

Theorem 3.3. Let $R>0, B=\{x \in E:\|x\| \leq R\}, U=\{x \in C(J, E):\|x\| \leq R\}$, and assume that:

(H1) $F: J \times E \rightarrow \mathcal{P}_{c p, p}(E)$ is a Carathéodory multi-valued map;

(H2) For each $R>0$, there exists a function $p \in L^{1}(J, E)$ such that

$$
\|F(t, u)\|_{\mathcal{P}}=\sup \{|v|: v(t) \in F(t, y)\} \leq p(t)
$$

for each $(t, y) \in J \times E$ with $|y| \geq R$, and

$$
\liminf _{R \rightarrow \infty} \frac{\int_{0}^{T} p(t) d t}{R}=\delta<\infty
$$

(H3) There exists a Carathéodory function $\psi: J \times[1,2 R] \rightarrow \mathbb{R}_{+}$such that

$$
\beta(F(t, M)) \leq \psi(t, \beta(M)) \text { a.e. } t \in J \text { and each } M \subset B ;
$$

(H4) The function $\varphi=0$ is the unique solution in $C(J,[1,2 R])$ of the inequality

$$
\begin{aligned}
\varphi(t) & \leq 2\left\{\frac{1}{\Gamma(r)} \int_{1}^{t}\left(\log \frac{t}{s}\right)^{r-1} \psi(s, \varphi(s)) \frac{d s}{s}\right. \\
& \left.+\frac{(\log t)^{r-1}}{(\log T)^{r-1}}\left[y_{T}+\frac{1}{\Gamma(r)} \int_{1}^{T}\left(\log \frac{T}{s}\right)^{r-1} \psi(s, \varphi(s)) \frac{d s}{s}\right]\right\} \text { for } t \in J .
\end{aligned}
$$


Then the BVP (1.1)-(1.2) has at least one solution in $C(J, B)$, provided that

$$
\delta<\frac{\Gamma(r+1)}{(\log T)^{r}}
$$

Proof. We wish to transform the problem (1.1)-(1.2) into a fixed point problem, so consider the multivalued operator

$$
\begin{aligned}
N(y) & =\left\{h \in C(J, \mathbb{R}): h(t)=\frac{1}{\Gamma(r)} \int_{1}^{t}\left(\log \frac{t}{s}\right)^{r-1} v(s) \frac{d s}{s}\right. \\
& \left.+\frac{(\log t)^{r-1}}{(\log T)^{r-1}}\left[y_{T}-\frac{1}{\Gamma(r)} \int_{1}^{T}\left(\log \frac{T}{s}\right)^{r-1} v(s) \frac{d s}{s}\right], \quad v \in S_{F, y}\right\} .
\end{aligned}
$$

Clearly, from Lemma 3.2, the fixed points of $N$ are solutions to (1.1)-(1.2). We shall show that $N$ satisfies the assumptions of Mönch's fixed point theorem. The proof will be given in several steps. First note that $\bar{U}=C(J, B)$.

Step 1: $N(y)$ is convex for each $y \in C(J, B)$.

Take $h_{1}, h_{2} \in N(y)$; then there exist $v_{1}, v_{2} \in S_{F, y}$ such that for each $t \in J$, we have

$$
\begin{aligned}
h_{i}(t) & =\frac{1}{\Gamma(r)} \int_{1}^{t}\left(\log \frac{t}{s}\right)^{r-1} v_{i}(s) \frac{d s}{s} \\
& +\frac{(\log t)^{r-1}}{(\log T)^{r-1}}\left[y_{T}-\frac{1}{\Gamma(r)} \int_{1}^{T}\left(\log \frac{T}{s}\right)^{r-1} v_{i}(s) \frac{d s}{s}\right]
\end{aligned}
$$

for $i=1,2$. Let $0 \leq d \leq 1$; then for each $t \in J$,

$$
\begin{aligned}
\left(d h_{1}+(1-d) h_{2}\right)(t) & =\frac{1}{\Gamma(r)} \int_{1}^{t}\left(\log \frac{t}{s}\right)^{r-1}\left[d v_{1}+(1-d) v_{2}\right] \frac{d s}{s} \\
& +\frac{(\log t)^{r-1}}{(\log T)^{r-1}}\left[y_{T}-\frac{1}{\Gamma(r)} \int_{1}^{T}\left(\log \frac{T}{s}\right)^{r-1}\left[d v_{1}+(1-d) v_{2}\right] \frac{d s}{s}\right] .
\end{aligned}
$$

Since $S_{F, y}$ is convex (because $F$ has convex values), we have

$$
d h_{1}+(1-d) h_{2} \in N(y) .
$$

Step 2: $N(M)$ is relatively compact for each compact $M \subset \bar{U}$.

Let $M \subset \bar{U}$ be a compact set and let $\left\{h_{n}\right\}$ be any sequence of elements of $N(M)$. We will show that $\left\{h_{n}\right\}$ has a convergent subsequence by using the ArzelàAscoli criterion of compactness in $C(J, B)$. Since $h_{n} \in N(M)$, there exist $y_{n} \in M$ and $v_{n} \in S_{F, y}$ such that

$$
\begin{aligned}
h_{n}(t) & =\frac{1}{\Gamma(r)} \int_{1}^{t}\left(\log \frac{t}{s}\right)^{r-1} v_{n}(s) \frac{d s}{s} \\
& +\frac{(\log t)^{r-1}}{(\log T)^{r-1}}\left[y_{T}-\frac{1}{\Gamma(r)} \int_{1}^{T}\left(\log \frac{T}{s}\right)^{r-1} v_{n}(s) \frac{d s}{s}\right]
\end{aligned}
$$


for $n \geq 1$. Using Theorem 2.3 and the properties of the Kuratowski measure of noncompactness, we have

$$
\begin{aligned}
\beta\left(\left\{h_{n}(t)\right\}\right) & \leq 2\left\{\frac{1}{\Gamma(r)} \int_{1}^{t} \beta\left(\left\{\left(\log \frac{t}{s}\right)^{r-1} \frac{v_{n}(s)}{s}: n \geq 1\right\}\right) d s\right. \\
& \left.+\frac{(\log t)^{r-1}}{(\log T)^{r-1}}\left[y_{T}+\frac{1}{\Gamma(r)} \int_{1}^{T} \beta\left(\left\{\left(\log \frac{T}{s}\right)^{r-1} \frac{v_{n}(s)}{s}: n \geq 1\right\}\right) d s\right]\right\} .
\end{aligned}
$$

On the other hand, since $M(s)$ is compact in $\mathrm{E}$, the set $\left\{v_{n}(s): n \geq 1\right\}$ is compact. Consequently, $\beta\left(\left\{v_{n}(s): n \geq 1\right\}\right)=0$ for a.e. $s \in J$. Furthermore,

$$
\beta\left(\left\{\left(\log \frac{t}{s}\right)^{r-1} \frac{v_{n}(s)}{s}\right\}\right)=\left(\log \frac{t}{s}\right)^{r-1} \frac{1}{s} \beta\left(\left\{v_{n}(s): n \geq 1\right\}\right)=0
$$

and

$$
\beta\left(\left\{\left(\log \frac{T}{s}\right)^{r-1} \frac{v_{n}(s)}{s}\right\}\right)=\left(\log \frac{T}{s}\right)^{r-1} \frac{1}{s} \beta\left(\left\{v_{n}(s): n \geq 1\right\}\right)=0
$$

for a.e. $t, s \in J$. Hence, from this and (3.7), $\left\{h_{n}(t): n \geq 1\right\}$ is relatively compact in $B$ for each $t \in J$. In addition, for each $t_{1}, t_{2} \in J$ with $t_{1}<t_{2}$, we have

$$
\begin{aligned}
\left|h_{n}\left(t_{2}\right)-h_{n}\left(t_{1}\right)\right| & =\mid \frac{1}{\Gamma(\alpha)} \int_{1}^{t_{1}}\left[\left(\log \frac{t_{2}}{s}\right)^{\alpha-1}-\left(\log \frac{t_{1}}{s}\right)^{\alpha-1}\right] \frac{v_{n}(s)}{s} d s \\
& +\frac{1}{\Gamma(\alpha)} \int_{t_{1}}^{t_{2}}\left(\log \frac{t_{2}}{s}\right)^{\alpha-1} \frac{v_{n}(s)}{s} d s \mid \\
& \leq \frac{p(t)}{\Gamma(\alpha)} \int_{1}^{t_{1}}\left[\left(\log \frac{t_{2}}{s}\right)^{\alpha-1}-\left(\log \frac{t_{1}}{s}\right)^{\alpha-1}\right] \frac{d s}{s} \\
& +\frac{p(t)}{\Gamma(\alpha)} \int_{t_{1}}^{t_{2}}\left(\log \frac{t_{2}}{s}\right)^{\alpha-1} \frac{d s}{s} .
\end{aligned}
$$

As $t_{1} \rightarrow t_{2}$, the right hand side of the above inequality tends to zero. This shows that $\left\{h_{n}: n \geq 1\right\}$ is equicontinuous. Consequently, $\left\{h_{n}: n \geq 1\right\}$ is relatively compact in $C(J, B)$.

Step 3: $N$ has a closed graph.

Let $y_{n} \rightarrow y_{*}, h_{n} \in N\left(y_{n}\right)$, and $h_{n} \rightarrow h_{*}$. We need to show that $h_{*} \in N\left(y_{*}\right)$. Now $h_{n} \in N\left(y_{n}\right)$ means that there exists $v_{n} \in S_{F, y}$ such that, for each $t \in J$,

$$
\begin{aligned}
h_{n}(t) & =\frac{1}{\Gamma(r)} \int_{1}^{t}\left(\log \frac{t}{s}\right)^{r-1} v_{n}(s) \frac{d s}{s} \\
& +\frac{(\log t)^{r-1}}{(\log T)^{r-1}}\left[y_{T}-\frac{1}{\Gamma(r)} \int_{1}^{T}\left(\log \frac{T}{s}\right)^{r-1} v_{n}(s) \frac{d s}{s}\right] .
\end{aligned}
$$


Consider the continuous linear operator $\theta: L^{1}(J, E) \rightarrow C(J, E)$ defined by

$$
\begin{aligned}
\theta(v)(t) \rightarrow h_{n}(t) & =\frac{1}{\Gamma(r)} \int_{1}^{t}\left(\log \frac{t}{s}\right)^{r-1} v_{n}(s) \frac{d s}{s} \\
& +\frac{(\log t)^{r-1}}{(\log T)^{r-1}}\left[y_{T}-\frac{1}{\Gamma(r)} \int_{1}^{T}\left(\log \frac{T}{s}\right)^{r-1} v_{n}(s) \frac{d s}{s}\right]
\end{aligned}
$$

Clearly, $\left\|h_{n}(t)-h_{*}(t)\right\| \rightarrow 0$ as $n \rightarrow \infty$. From Lemma 2.4 it follows that $\theta \circ S_{F}$ is a closed graph operator. Moreover, $h_{n}(t) \in \theta\left(S_{F, y_{n}}\right)$. Since $y_{n} \rightarrow y$, Lemma 2.4 implies

$$
\begin{aligned}
h(t) & =\frac{1}{\Gamma(r)} \int_{1}^{t}\left(\log \frac{t}{s}\right)^{r-1} v(s) \frac{d s}{s} \\
& +\frac{(\log t)^{r-1}}{(\log T)^{r-1}}\left[y_{T}-\frac{1}{\Gamma(r)} \int_{1}^{T}\left(\log \frac{T}{s}\right)^{r-1} v(s) \frac{d s}{s}\right] .
\end{aligned}
$$

Step 4: $M$ is relatively compact in $C(J, B)$.

Suppose $M \subset \bar{U}, M \subset \operatorname{conv}(\{0\} \cup N(M))$, and $\bar{M}=\bar{C}$ for some countable set $C \subset M$. Using an argument similar to the one used in Step 2 shows that $N(M)$ is equicontinuous. Then, since $M \subset \operatorname{conv}(\{0\} \cup N(M))$, we see that $M$ is equicontinuous as well. To apply the Arzelà-Ascoli theorem, it remains to show that $M(t)$ is relatively compact in $E$ for each $t \in J$. Since $C \subset M \subset \operatorname{conv}(\{0\} \cup N(M))$ and $C$ is countable, we can find a countable set $H=\left\{h_{n}: n \geq 1\right\} \subset N(M)$ with $C \subset \operatorname{conv}(\{0\} \cup H)$. Then, there exist $y_{n} \in M$ and $v_{n} \in S_{F, y_{n}}$ such that

$$
\begin{aligned}
h_{n}(t) & =\frac{1}{\Gamma(r)} \int_{1}^{t}\left(\log \frac{t}{s}\right)^{r-1} v_{n}(s) \frac{d s}{s} \\
& +\frac{(\log t)^{r-1}}{(\log T)^{r-1}}\left[y_{T}-\frac{1}{\Gamma(r)} \int_{1}^{T}\left(\log \frac{T}{s}\right)^{r-1} v_{n}(s) \frac{d s}{s}\right] .
\end{aligned}
$$

Since $M \subset C \subset \operatorname{conv}(\{0\} \cup H))$, from the properties of the Kuratowski measure of noncompactness, we have

$$
\beta(M(t)) \leq \beta(C(t)) \leq \beta(H(t))=\beta\left(\left\{h_{n}(t): n \geq 1\right\}\right) .
$$


Using (3.7) and the fact that $v_{n}(s) \in M(s)$, we obtain

$$
\begin{aligned}
\beta(M(t)) & \leq 2\left\{\frac{1}{\Gamma(r)} \int_{1}^{t} \beta\left(\left\{\left(\log \frac{t}{s}\right)^{r-1} \frac{v_{n}(s)}{s}: n \geq 1\right\}\right) d s\right. \\
& \left.+\frac{(\log t)^{r-1}}{(\log T)^{r-1}}\left[y_{T}+\frac{1}{\Gamma(r)} \int_{1}^{T} \beta\left(\left\{\left(\log \frac{T}{s}\right)^{r-1} \frac{v_{n}(s)}{s}: n \geq 1\right\}\right) d s\right]\right\} \\
& \leq 2\left\{\frac{1}{\Gamma(r)} \int_{1}^{t}\left(\log \frac{t}{s}\right)^{r-1} \beta(M(s)) \frac{d s}{s}\right. \\
& \left.+\frac{(\log t)^{r-1}}{(\log T)^{r-1}}\left[y_{T}+\frac{1}{\Gamma(r)} \int_{1}^{T}\left(\log \frac{T}{s}\right)^{r-1} \beta(M(s)) \frac{d s}{s}\right]\right\} \\
& \leq 2\left\{\frac{1}{\Gamma(r)} \int_{1}^{t}\left(\log \frac{t}{s}\right)^{r-1} \psi(s, \beta(M(s))) \frac{d s}{s}\right. \\
& \left.+\frac{(\log t)^{r-1}}{(\log T)^{r-1}}\left[y_{T}+\frac{1}{\Gamma(r)} \int_{1}^{T}\left(\log \frac{T}{s}\right)^{r-1} \psi(s, \beta(M(s))) \frac{d s}{s}\right]\right\} .
\end{aligned}
$$

We also have that the function $\varphi$ given by $\varphi(t)=\beta(M(t))$ belongs to $C(J,[1,2 R])$. Consequently, by $(H 4), \varphi=0$; that is, $\beta(M(t))=0$ for all $t \in J$. Now, by the Arzelà-Ascoli theorem, $M$ is relatively compact in $C(J, B)$.

Step 5: Let $h \in N(y)$ with $y \in U$. We claim that $N(U) \subset U$. If this were not the case, then in view of $(H 2)$, there exists functions $v \in S_{F, y}$ and $p \in L^{1}(J, E)$ such that

$$
\begin{aligned}
h(t) & =\frac{1}{\Gamma(r)} \int_{1}^{t}\left(\log \frac{t}{s}\right)^{r-1} v(s) \frac{d s}{s} \\
& +\frac{(\log t)^{r-1}}{(\log T)^{r-1}}\left[y_{T}-\frac{1}{\Gamma(r)} \int_{1}^{T}\left(\log \frac{T}{s}\right)^{r-1} v(s) \frac{d s}{s}\right],
\end{aligned}
$$

and

$$
\begin{aligned}
R<\|N(y)\|_{\mathcal{P}} & \leq \frac{1}{\Gamma(r)} \int_{1}^{t}\left(\log \frac{t}{s}\right)^{r-1}|v(s)| \frac{d s}{s} \\
& +\frac{(\log t)^{r-1}}{(\log T)^{r-1}}\left[\left|y_{T}\right|+\frac{1}{\Gamma(r)} \int_{1}^{T}\left(\log \frac{T}{s}\right)^{r-1}|v(s)| \frac{d s}{s}\right] \\
& \leq \frac{(\log T)^{r}}{\Gamma(r+1)} \int_{1}^{t} p(s) d s+\frac{(\log T)^{r}}{\Gamma(r+1)} \int_{1}^{T} p(s) d s \\
& \leq 2 \frac{(\log T)^{r}}{\Gamma(r+1)} \int_{1}^{t} p(s) d s .
\end{aligned}
$$

Dividing both sides by $R$ and taking the $\lim \inf$ as $R \rightarrow \infty$, we have

$$
2\left[\frac{(\log T)^{r}}{\Gamma(r+1)}\right] \delta \geq 1
$$


which contradicts (3.6). Hence, $N(U) \subset U$.

As a consequence of Steps 1-5 and Mönch's Theorem (Theorem 2.7 above), $N$ has a fixed point $y \in C(J, B)$ that in turn is a solution of problem (1.1)-(1.2).

\section{An example}

We conclude this paper with an example to illustrate our main result, namely, Theorem 3.3 above.

Consider the fractional differential inclusion

$$
\begin{gathered}
{ }^{H} D^{\alpha} y(t) \in F(t, y(t)), \text { for a.e. } t \in J=[1, e], 0<\alpha \leq 1, \\
y(1)=0, y(e)=1 .
\end{gathered}
$$

Here, $F:[1, e] \times \mathbb{R} \rightarrow \mathcal{P}(\mathbb{R})$ is a multivalued map satisfying

$$
F(t, y)=\left\{v \in \mathbb{R}: f_{1}(t, y) \leq v \leq f_{2}(t, y)\right\},
$$

where $f_{1}, f_{2}:[1, e] \times \mathbb{R} \rightarrow \mathbb{R}, f_{1}(t, \cdot)$ is lower semi-continuous (i.e., the set $\{y \in \mathbb{R}:$ $\left.f_{1}(t, y)>\mu\right\}$ is open for each $\mu \in \mathbb{R}$ ), and $f_{2}(t, \cdot)$ is upper semi-continuous (i.e., the set the set $\left\{y \in \mathbb{R}: f_{2}(t, y)<\mu\right\}$ is open for each $\left.\mu \in \mathbb{R}\right)$. We assume that there is a function $p \in L^{1}(J, \mathbb{R})$ such that

$$
\begin{aligned}
\|F(t, u)\|_{\mathcal{P}} & =\sup \{|v|, v(t) \in F(t, y)\} \\
& =\max \left(\left|f_{1}(t, y)\right|,\left|f_{2}(t, y)\right|\right\} \leq p(t), t \in[1, e], y \in \mathbb{R} .
\end{aligned}
$$

It is clear that $F$ is compact and convex valued, and is upper semi-continuous.

Choose $C(s)$ to be the space of linear functions and choose $\varphi(t)=\beta(C(t))$ such that

$$
\beta(u(s))=\frac{u(s)}{2}
$$

with

$$
u(s)=a s, a>0, \frac{2}{a} \leq s \leq \frac{4 R}{a} .
$$

For $(t, y) \in J \times \mathbb{R}$ with $|y| \geq R$, we have

$$
\liminf _{R \rightarrow \infty} \frac{\int_{0}^{e} p(t) d t}{R}=\delta<\infty .
$$

Finally, we assume that there exists a Carathéodory function $\psi: J[1,2 R] \rightarrow \mathbb{R}_{+}$such that

$$
\beta(F(t, M)) \leq \psi(t, \beta(M)) \text { a.e. } t \in J \text { and each } M \subset B=\{x \in \mathbb{R}:|x| \leq R\},
$$

and $\varphi=0$ is the unique solution in $C(J,[1,2 R])$ of the inequality

$$
\begin{aligned}
\varphi(t) & \leq 2\left\{\frac{1}{\Gamma(r)} \int_{1}^{t}\left(\log \frac{t}{s}\right)^{r-1} \psi(s, \varphi(s)) \frac{d s}{s}\right. \\
& \left.+(\log t)^{r-1}\left[1+\frac{1}{\Gamma(r)} \int_{1}^{e}\left(\log \frac{e}{s}\right)^{r-1} \psi(s, \varphi(s)) \frac{d s}{s}\right]\right\} .
\end{aligned}
$$

for $t \in J$. 
Since all the conditions of Theorem 3.3 are satisfied, problem (4.1)-(4.2) has at least one solution $y$ on $[1, e]$.

\section{References}

[1] Agarwal, R.P., Benchohra, M., Seba, D., An the application of measure of noncompactness to the existence of solutions for fractional differential equations, Results Math. 55(2009), 221-230.

[2] Ahmed, B., Ntouyas, S.K., Initial value problems for hybrid Hadamard fractional equations, Electron. J. Differential Equations, 2014(2014), no. 161, 1-8.

[3] Akhmerov, R.R., Kamenski, M.I., Patapov, A.S., Rodkina, A.E., Sadovski, B.N., Measures of Noncompactness and Condensing Operators (Translated from the 1986 Russian original by A. Iacop), Operator theory: Advances and Applications, 55, Birkhäuser Verlag, Bassel, 1992.

[4] Aubin, J.P., Cellina, A., Differential Inclusions, Springer-Verlag, Berlin-Heidelberg, New York, 1984.

[5] Aubin, J.P., Frankowska, H., Set-Valued Analysis, Birkhäuser, Boston, 1990.

[6] Banas, J., Goebel, K., Measure of Noncompactness in Banach Spaces, Lecture Notes in Pure and Applied Mathematics, Vol. 60, Dekker, New York.

[7] Benchohra, M., Henderson, J., Seba, D., Measure of noncompactness and fractional differential equations in Banach spaces, Commun. Appl. Anal., 12(2008), 419-428.

[8] Benchohra, M., Henderson, J., Seba, D., Boundary value problems for fractional differential inclusions in Banach space, Fract. Differ. Calc., 2(2012), 99-108.

[9] Benchohra, M., Nieto, J.J., Seba, D., Measure of noncompactness and fractional and hyperbolic partial fractional differential equations in Banach space, Panamer. Math. J., 20(2010), 27-37.

[10] Benhamida, W., Graef, J.R., Hamani, S., Boundary value problems for fractional differential equations with integral and anti-periodic conditions in a Banach space, (to appear).

[11] Butzer, P.L., Kilbas, A.A., Trujillo, J.J., Composition of Hadamard-type fractional integration operators and the semigroup property, J. Math. Anal. Appl., 269(2002), 387-400.

[12] Butzer, P.L., Kilbas, A.A., Trujillo, J.J., Fractional calculus in the Mellin setting and Hadamard-type fractional integrals, J. Math. Anal. Appl., 269(2002), 1-27.

[13] Butzer, P.L., Kilbas, A.A., Trujillo, J.J., Mellin transform analysis and integration by parts for Hadamard-type fractional integrals, J. Math. Anal. Appl., 270(2002), 1-15.

[14] Castaing, C., Valadier, M., Convex Analysis and Measurable Multifunctions, Lecture Notes in Mathematics 580, Springer-Verlag, Berlin-Heidelberg-New York, 1977.

[15] Deimling, K., Multivalued Differential Equations, De Gruyter, Berlin-New York, 1992.

[16] Hadamard, J., Essai sur l'etude des fonctions donnees par leur development de Taylor, J. Mat. Pure Appl., 8(1892), 101-186.

[17] Heinz, H.P., On the behavior of measure of noncompactness with respect of differentiation and integration of vector-valued function, Nonlinear. Anal, 7(1983), 1351-1371.

[18] Hilfer, R., Applications of Fractional Calculus in Physics, World Scientific, Singapore, 2000 . 
[19] Kilbas, A.A., Srivastava, H.M., Trujillo, J.J., Theory and Applications of Fractional Differential Equations, North-Holland Mathematics Studies, 204, Elsevier, Amsterdam, 2006.

[20] Lasota, A., Opial, Z., An application of the Kakutani-Ky Fan theorem in the theory of ordinary differential equation, Bull. Accd. Pol. Sci. Ser. Sci. Math. Astronom. Phys., 13(1965), 781-786.

[21] Momani, S.M., Hadid, S.B., Alawenh, Z.M., Some analytical properties of solutions of diifferential equations of noninteger order, Int. J. Math. Math. Sci., 2004(2004), 697-701.

[22] O'Regan, D., Precup, R., Fixed point theorems for set-valued maps and existence principles for integral inclusions, J. Math. Anal. Appl., 245(2000), 594-612.

[23] Podlubny, I., Fractional Differential Equation, Academic Press, San Diego, 1999.

[24] Thiramanus, P., Ntouyas, S.K., Tariboon, J., Existence and uniqueness results for Hadamard-type fractional differential equations with nonlocal fractional integral boundary conditions, Abstr. Appl. Anal., (2014), Art. ID 902054, 9 pp.

John R. Graef

Department of Mathematics

University of Tennessee at Chattanooga

Chattanooga, TN 37403-2504, USA

e-mail: John-Graef@utc.edu

Nassim Guerraiche

Laboratoire des Mathématiques Appliqués et Pures

Université de Mostaganem

B.P. 227, 27000, Mostaganem, Algerie

e-mail: hamani_samira@yahoo.fr

Samira Hamani

Laboratoire des Mathématiques Appliqués et Pures

Université de Mostaganem

B.P. 227, 27000, Mostaganem, Algerie

e-mail: nassim.guerraiche@univ-mosta.dz 